Corrigendum

\title{
Corrigendum to "SARC018_SPORE02: Phase II Study of Mocetinostat Administered with Gemcitabine for Patients with Metastatic Leiomyosarcoma with Progression or Relapse following Prior Treatment with Gemcitabine-Containing Therapy"
}

\author{
Edwin Choy $\mathbb{D}^{1}{ }^{1}$ Karla Ballman, ${ }^{2}$ James Chen, ${ }^{3}$ Mark A. Dickson, ${ }^{4}$ Rashmi Chugh $\mathbb{D}^{5}{ }^{5}$ \\ Suzanne George, ${ }^{6}$ Scott Okuno $\mathbb{D}^{7}{ }^{7}$ Raphael Pollock, ${ }^{8}$ Rajiv M. Patel, ${ }^{9}$ Antje Hoering, ${ }^{10}$ \\ and Shreyaskumar Patel iD $^{11}$ \\ ${ }^{1}$ Massachusetts General Hospital, Division of Hematology Oncology, 55 Fruit Street, Boston, MA 02114, USA \\ ${ }^{2}$ Weill Cornell Medicine Healthcare Policy and Research, 402 East 67th Street, LA-225, New York, NY 10065, USA \\ ${ }^{3}$ The Ohio State University Comprehensive Cancer Center, 1800 Cannon Drive, 250 Lincoln Tower, Columbus, OH 43210, USA \\ ${ }^{4}$ Memorial Sloan Kettering Cancer Center and Weill Cornell Medical College, 300 E. 66th Street, New York, NY 10065, USA \\ ${ }^{5}$ University of Michigan Comprehensive Cancer Center, 1500 E. Medical Center Drive, Rm C-407 MIB/SPC 5843, Ann Arbor, \\ MI 48109, USA \\ ${ }^{6}$ Dana-Farber Cancer Institute, Center for Sarcoma and Bone Oncology, 450 Brookline Ave, D-1212, Brookline, MA 02215, USA \\ ${ }^{7}$ Mayo Clinic, 200 First St. SW, Rochester, MN 55905, USA \\ ${ }^{8}$ Ohio State University Wexner Medical Center, 410 West 10th Avenue, N924 Doan Hall, Columbus, OH 43206, USA \\ ${ }^{9}$ Michigan Medicine, Department of Pathology, 1301 Catherine Street, SPC 5602, 3261G Medical Science I, Ann Arbor, \\ MI 48109-5602, USA \\ ${ }^{10}$ Cancer Research and Biostatistics, 1730 Minor Ave, Suite 1900, Seattle, WA 98101, USA \\ ${ }^{11}$ MD Anderson Cancer Center, Sarcoma Medical Oncology, 1400 Holcombe Blvd, Unit 450, Houston, TX 77030, USA
}

Correspondence should be addressed to Edwin Choy; echoy@mgh.harvard.edu

Received 10 July 2019; Accepted 15 July 2019; Published 27 August 2019

Copyright (c) 2019 Edwin Choy et al. This is an open access article distributed under the Creative Commons Attribution License, which permits unrestricted use, distribution, and reproduction in any medium, provided the original work is properly cited.

In the article titled "SARC018_SPORE02: Phase II Study of Mocetinostat Administered with Gemcitabine for Patients with Metastatic Leiomyosarcoma with Progression or Relapse following Prior Treatment with Gemcitabine-Containing Therapy" [1], the second author, Dr. Ballman, was paid by Lilly and only recently realized that gemcitabine is a Lilly product and apologizes for not declaring this sooner.

\section{References}

[1] E. Choy, K. Ballman, J. Chen et al., "SARC018_SPORE02: phase II study of mocetinostat administered with gemcitabine for patients with metastatic leiomyosarcoma with progression or relapse following prior treatment with gemcitabine-containing therapy," Sarcoma, vol. 2018, Article ID 2068517, 9 pages, 2018. 


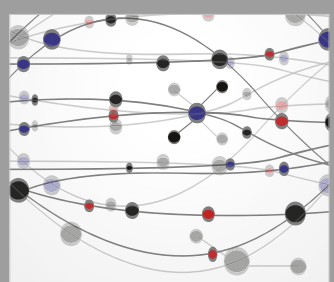

The Scientific World Journal
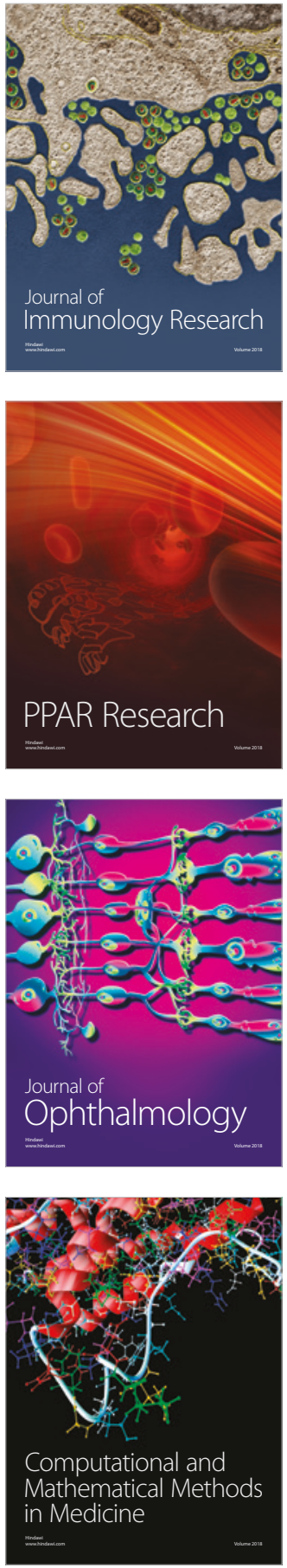

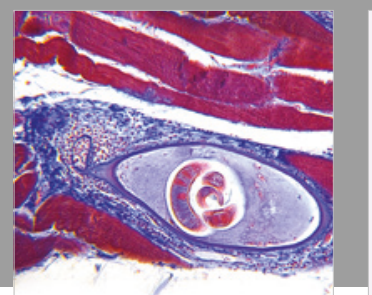

Gastroenterology Research and Practice

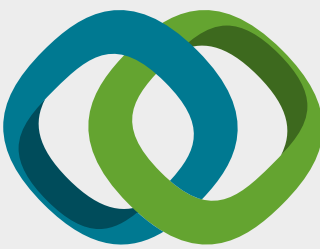

\section{Hindawi}

Submit your manuscripts at

www.hindawi.com
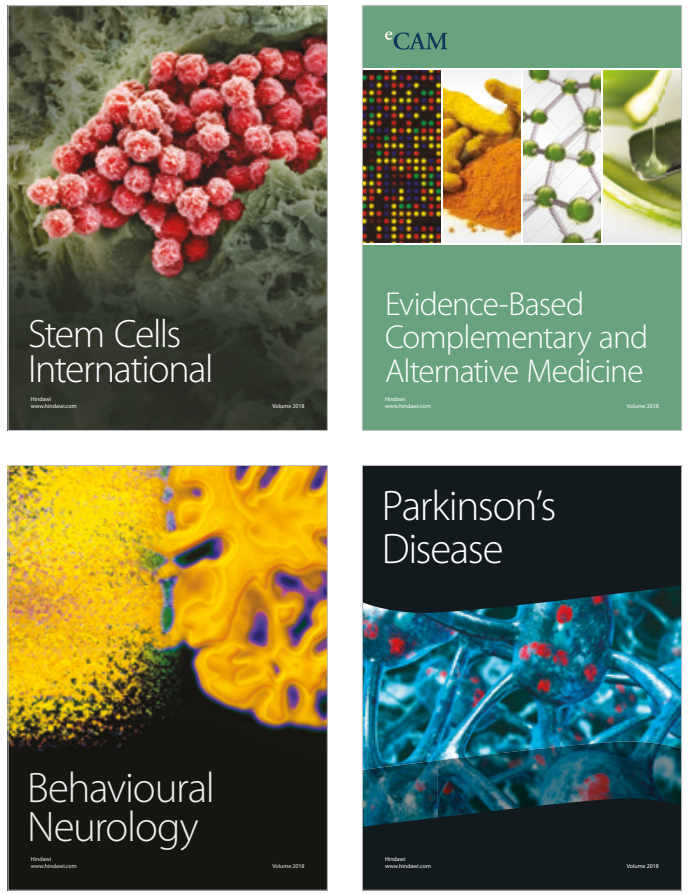

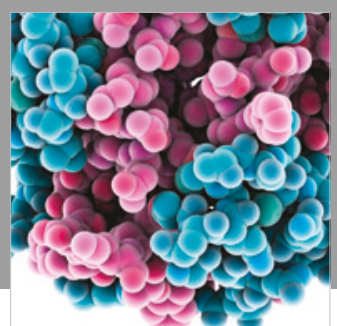

ournal of

Diabetes Research

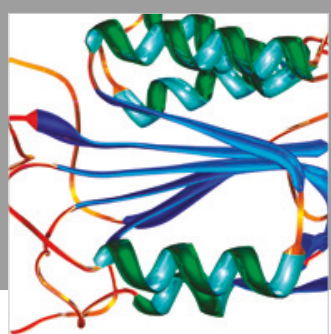

Disease Markers
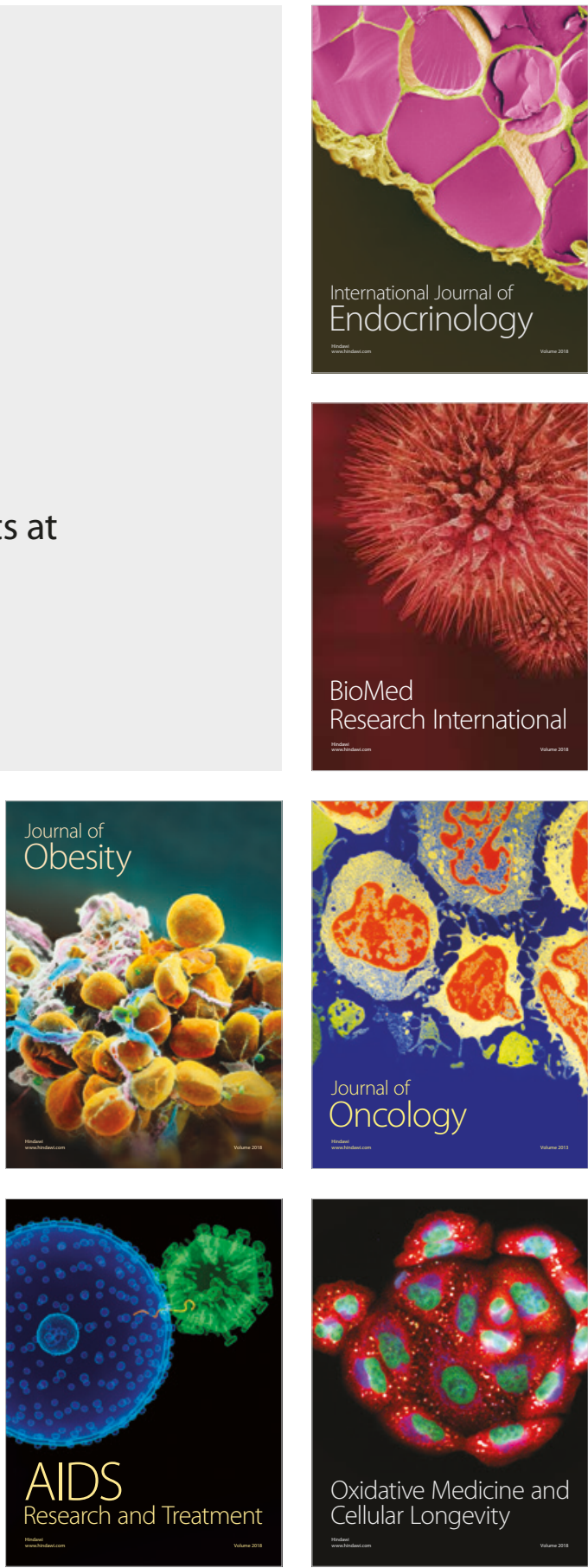